\title{
Intraday variability of the polarization vector in AGN S5 0716+714
}

\author{
E. Shablovinskaya and V. Afanasiev \\ Special Astrophysical Observatory of the Russian Academy of Science, Nizhnii \\ Arkhyz,369167, Russia,(E-mail: e.shablie@yandex.com,vafan@sao.ru)
}

Received: July 28, 2019; Accepted: September 28, 2019

\begin{abstract}
The bright radio source S5 $0716+714$, which is usually classified as a BL Lac object, is one of the most intensively studied blazar. S5 $0716+714$ demonstrates extremely peculiar properties, such as the shortest time-scale of optical and polarimetric variations observed in blazars. In the given talk, we present the results of a 8-h polarimetric monitoring of S5 $0716+714$ with a $\sim 70$-sec resolution carried out using the 6-m telescope BTA of the SAO RAS. The observation data analysis reveals the variability both in total and polarized light on the 1.5-hour timescales that specifies the size of the unresolved emitting region. The numerical model of polarization in jet with helical structure of magnetic field is suggested, and fitting the model reveals a magnetic field precession with a period of about 15 days.
\end{abstract}

Key words: BL Lacertae objects: individual: S5 0716+714 - polarization galaxies: jets - galaxies: distances and redshifts

\section{Introduction}

BL Lac type objects or blazars ${ }^{1}$ are a special type of active galactic nucleus (AGN) with the jet directed almost toward the observer. Because of such orientation, the synchrotron (non-thermal) component formed in the jet makes a very large contribution in the blazar optical radiation, and short-term brightness and polarization variations are observed (the polarization degree in flashes is up 40\% and higher, see e.g. polarization light curve of PKS 1510-089 in the paper by Marscher et al. (2010)).

S5 $0716+714$ is considered to be a typical BL Lac object. It shows flat power-law spectrum $\left(\alpha \geq-0.5, S_{\nu} \propto \nu_{\alpha}\right.$ in the radio band), as well as intraday variability in all spectral ranges: from 8-12 hours in radio band $(6 \mathrm{~cm}$, Gorshkov et al., 2011; Gorshkov et al., 2011) to 2.2-3.2 min flares in X-rays (Pryal et al., 2015). The observed polarization is also variable as within the night (e.g. Impey et al., 2000; Amirkhanyan, 2006), and on the scale of tens of days (e.g. Larionov et al., 2013). However, the increase in the polarization is not correlated with optical flashes.

\footnotetext{
${ }^{1}$ Though the terms "BL Lacs" and "blazars" are not equal to each other, within this paper we would assume it interchangeably.
} 
It is assumed the plasma motion in a relativistic jet is responsible for the observed object variability. In this regard, this work is devoted to the detection of the polarization vector motion associated with the plasma, as well as the construction of a model of polarization changes within the night.

\section{S5 $0716+714-$ what are you?}

The biggest question related to object S5 $0716+714$ is the redshift estimation. The lines weakness and their small equivalent width in the blazar spectra are normal since the contribution of the non-thermal jet component is too large. However, in the case of S5 $0716+714$, no details up to $0.3 \%$ are detected in the spectrum that is also mentioned in the work by Nilsson et al. (2008).

In February the spectroscopic observations of S5 $0716+714$ were carried out at the BTA telescope using the SCORPIO-2 device (Afanasiev et al., 2017) while the object was in an almost record low activity state, when its luminosity was about $14.8 \mathrm{mag}$ in $R$ band $^{2}$. Three $600 \mathrm{sec}$ exposure spectra were obtained with a VPHG940@600 grating. The slit was oriented so that the spectrum of the neighbouring star was observed simultaneously with the object. In Fig. 1 the object and the star spectra are presented in the range $3700-8200 \AA$ in residual intensities. As it can be seen from the figure, in the spectrum of S5 $0716+714$ there is no detail, despite low activity state, except noted atmospheric lines common to the object and the stars.

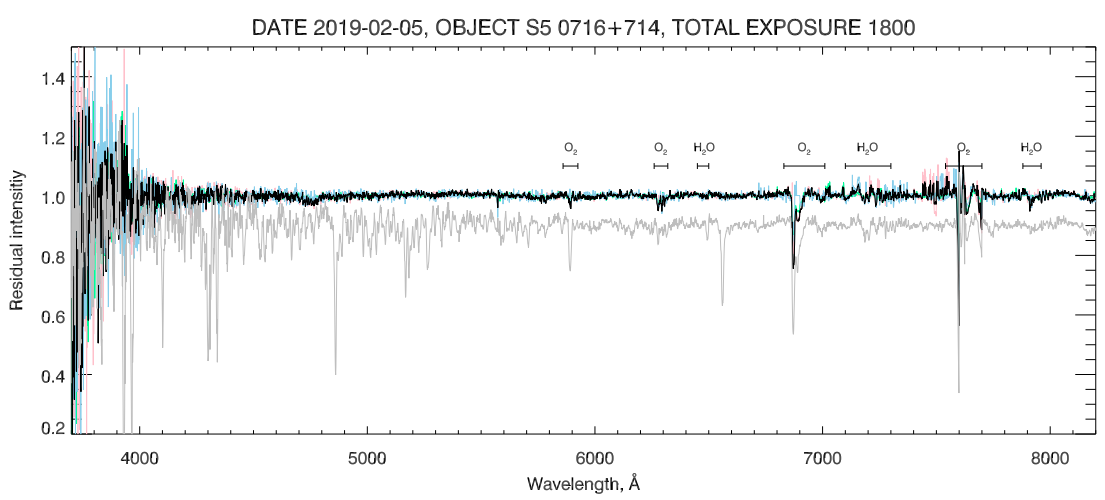

Figure 1. The spectrum of S5 $0716+714$ in residual intensities obtained while the object was in inactive state: the average S5 $0716+714$ spectrum is plotted in black and the comparison star is in gray. The telluric $\mathrm{O}_{2}$ and $\mathrm{H}_{2} \mathrm{O}$ lines are marked.

${ }^{2}$ According to the monitoring provided by Saint Petersburg State University observatory http://vo.astro.spbu.ru/en/program. 
Also, the spectrum obtained at the BTA with the SCORPIO device in 2010 is shown (Fig. 2, upper panel). The object was in a brighter state ( 13 mag in the $R$ band), but its lines are still not visible. The spectrum contains interstellar molecular bands (DIBs) and H\&K CaII lines (Fig. 2, bottom panel). The H\&K CaII equivalent width is $W_{\lambda}=185 \pm 6 \mathrm{~m} \AA$, and according to the calibration (Beers et al., 1990) is insufficient for an extragalactic object and corresponds to a distance less than $1 \mathrm{kpc}$.

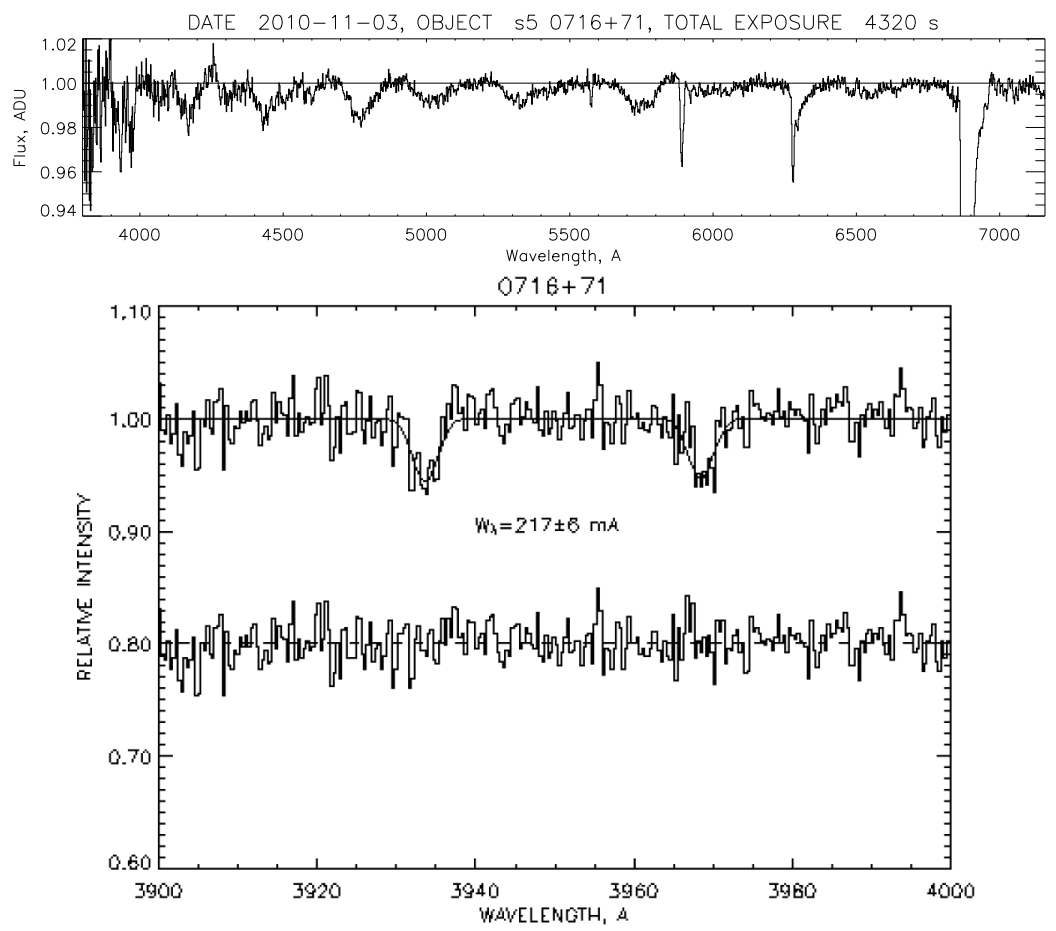

Figure 2. Upper panel: the spectrum of S5 0716+714 in residual intensities with DIBs. Bottom panel: equivalent width of Galactic H\&K CaII in S5 0716+714 spectrum.

Other indirect methods were also used to find the S5 $0716+714$ redshift. The first try was described in the paper (Stickel et al., 1993). Two weak galaxies (at distances of $27^{\prime \prime}$ and $55^{\prime \prime}$ from the source - $0.11 \mathrm{Mpc}$ and $0.23 \mathrm{Mpc}$ for $z \sim 0.3$, respectively) with redshifts of $\sim 0.26$ were found and it was concluded the source $z$ is close to this value. However, the assumption about the galaxy cluster is not confirmed by X-ray data (see e.g. X-ray galaxy cluster surveys by Romer et al. (2000); Burenin et al. (2007)). Attempts were also made to detect the host galaxy. In the works (Nilsson et al., 2008; Stadnik \& Romani, 2014) the PSF fitting was used giving an inconsistent result: $z \sim 0.3$ and 0.127 respectively. 
It is important to note that in the BL Lac type objects survey made by HST (Urry et al., 2000) no evidence of the host galaxy was detected in S5 0716+714 despite the inactive state $(14.18 \mathrm{mag}$ in $R$ ), and a restriction on the redshift $z>0.5$ was given.

Another specific feature of the S5 0716+714 object is its brightness. Based on the HST survey, the maximum brightness difference between the core and the host is $4 \mathrm{mag}$ on average for the blazar sample. Yet for S5 $0716+714$ it is up 7 mag. The reason for such a tremendous brightness remains unclear.

The above-mentioned features of the object doubt on its extragalactic nature. The observed synchrotron radiation is typical for all accreting systems both active nuclei and compact galactic systems, for example, low-mass X-ray binaries. In fact, when the system is oriented so that the relativistic jet points toward the observer and such details are absent as any marks of the surroundings or spectral features it is hard or even impossible to unambiguously answer the question about its type. This fact makes us free to raise an issue if S5 0716+714 is an extragactic object or it is just a system with a jet in the Galaxy, and we are looking for a critical test.

Within the frames of this work, we will consider the object S5 0716+714 belongs to the BL Lac type, although the conclusions we give about the jet radiation can be applied to sources of a different nature.

\section{Polarimetric observations}
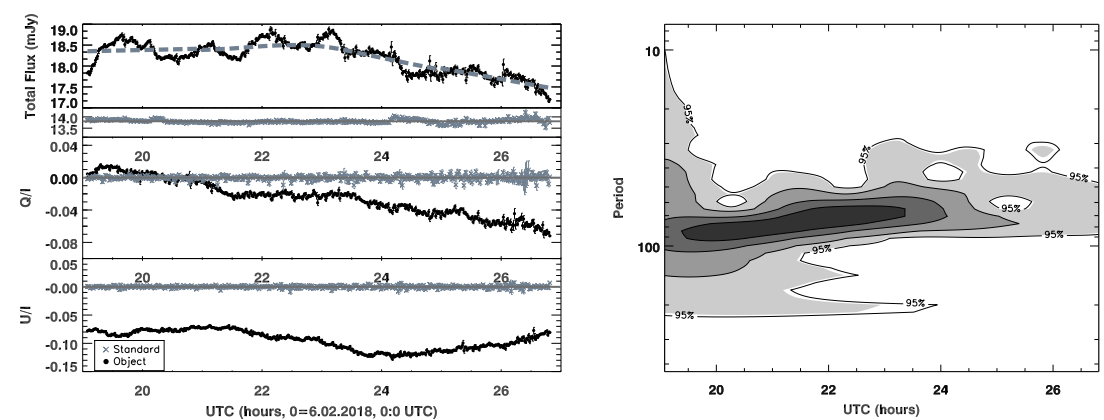

Figure 3. Left: the variations of the total flux and the Stokes parameters $Q$ and $U$ during the night. The observations started at 19:08 on 2018 February 2 (UTC). Right: The magnitude of the wavelet transform for the total flux.

In February 2018, we conducted 8-hour polarimetric monitoring of the S5 $0716+714$ object with a 1-minute temporal resolution at the BTA telescope with the SCORPIO-2 device. There are two important methodic features: 
(i) the observations of the object and the comparison star at a distance of $\sim 1^{\prime}$ occur simultaneously. Since the star is photometrically constant and has zero polarization (Amirkhanyan, 2006), it is possible to minimize the transmission variations and the atmospheric depolarization;

(ii) the double Wollaston prism (Geyer et al., 1996; Oliva, 1997) was used as a polarization analyzer to measure both linear polarization parameters - the Stokes parameters $Q$ and $U$ - simultaneously.

The obtained accuracy was about $0.005 \mathrm{mag}$ for photometry and $0.1 \%$ for polarimetry.

As a result, an 8-hour data series was obtained (Fig. 3, left), where significant changes in both flux and polarization are observed. To study the total flux variability the long-period trend was approximated by the robust smoothing 2degree polynomial function ${ }^{3}$ and subtracted. The wavelet analysis (Grossmann \& Morlet, 1984) provided a period of $77 \pm 10 \mathrm{~min}$ of rapid variations (Fig. 3, right).

To study the variability of the $Q$ and $U$ parameters, they were plotted on the $Q U$-plane (Fig. 4). During the night the polarization vector changed its direction several times about every 1.5-3 hours, and the changes are perpendicular to the jet direction. Therefore, "loops" and "arcs" are observed on the $Q U$-plane. Moreover, the period of the polarization vector direction and the total flux changes are similar. Indeed, if we assume that the observed motion of the polarization vector is caused by the plasma motion in the jet, then the polarization vector rotation will be due to the plasma changing direction and, consequently, a change in the Doppler amplification of its brightness for the observer.

\section{Data comparison and model}

Similar results were found for S5 $0716+714$ in earlier papers. Thus, the discovery that the polarization of an object changes on the $Q U$-plane not stochastically but along the definite trajectories was made in the work by Impey et al. (2000). However, the time sets duration was not enough to investigate the way the polarization vector change.

Furthermore, in the case of the object BL Lacertae (ancestor of the class blazars), a similar picture was found. We have examined polarimetric data, obtained in the paper by Covino et al. (2015) as the angle and degree of polarization separately, on the $Q U$-plane, where we also obtained the rotation of the polarization vector on the scales of several hours. Besides, for BL Lac, such a conclusion was made earlier in the almost forgotten work Moore et al. (1982): BL Lac showed the rotation of the polarization vector on the scales of hours in more than 7-day monitoring.

\footnotetext{
${ }^{3}$ The function was close to 6 -degree polynomial one.
} 


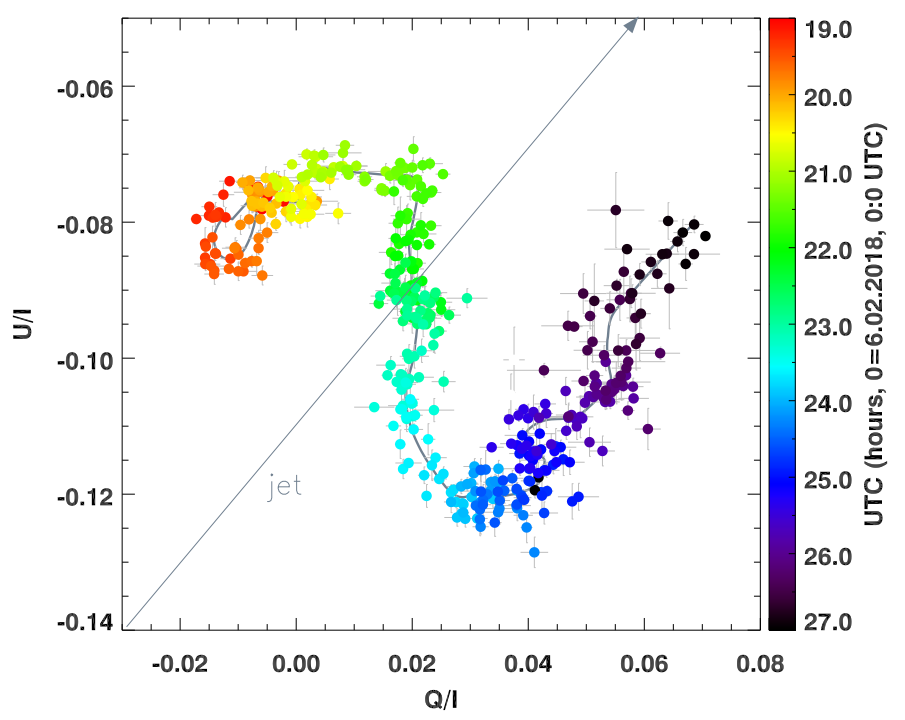

Figure 4. The variations of the normalized Stokes parameters $Q$ and $U$ during the night on the $Q U$-diagram.

Also, the rotation of the polarization vector along the arcs was found in the radio band in observations of blazar CTA 102 (Li et al., 2018). It was assumed there that the plasma in the jet should rotate along helical trajectories. On the other hand, according to the commonly used model (Marscher et al., 2008), it is known that the optical synchrotron radiation in the AGN jet is formed at a distance of $\sim 10^{-3}$ pc from the central source, where the magnetic field has a helical structure.

Based on these principles, we constructed a geometric model of polarization change in the jet helical magnetic field (in more details - Shablovinskaya \& Afanasiev, 2019). However, the numerical model of polarization change showed that it is impossible to describe the observed motion of the polarization vector by a stable configuration of the field. An important feature of our model was the field precession as an additional kinematic component. Then the rotation of the polarization vector on the $Q U$-plane observed during the 8-hour monitoring is described by the motion of the plasma in a helical magnetic field precessing with a period of $\sim 15$ days. Moreover, the linear size of the region where the optical polarization is formed is associated with the time of its variability, that is, they are about 1.5 lt hours or 10 a.u. Comparison of the model with observational data is shown in Fig. 5, where $3 \sigma$ confidence area of the smoothed polarization vector rotation is plotted light grey. The model fits the data good except the 


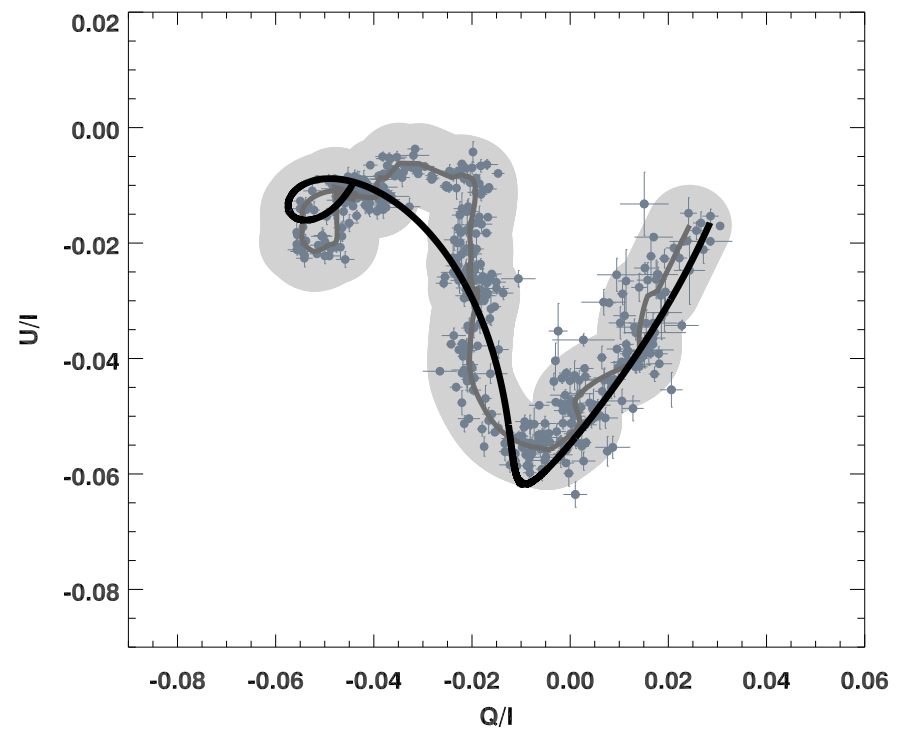

Figure 5. The model of linear polarization in the $Q U$-plane in the case of jet precession. The observational data are plotted with grey dots with error bars. The $3 \sigma$ confidence area is coloured light grey.

region between 21 and 22 hours. We attribute this divergence to the physical processes (radiation transfer and etc.) that we have not considered as our model describes the geometry of the plasma motion, and this neglect does not affect our quantitative results.

\section{Conclusions}

During the polarimetric monitoring of the S5 $0716+714$ object we obtained the following results.

(i) We found the variability of the total $(\Delta=0.04 \mathrm{mag})$ and polarized $(\Delta=7 \%)$ fluxes on a time-scale $\sim 1.5$ hours.

(ii) We discovered the specific pattern of the polarization vector on the $Q U$-plane - arches and loops.

(iii) The estimation of the linear size of the field identifying with the emitting region $1.5 \cdot 10^{-5} \mathrm{pc}$, or 10 a.u. at $\sim 10^{-3} \mathrm{pc}$ from the central $\mathrm{BH}$.

(iv) The polarization vector rotations mark the magnetic field precessing with the 15 days period.

(v) The similar pattern was found in other papers and also for BL Lac. 
Acknowledgements. We sincerely thank V. R. Amirkhanyan for valuable discussions and useful remarks. The results of observations were obtained with the $6-\mathrm{m}$ BTA telescope of the Special Astrophysical Observatory Academy of Sciences, operating with financial support from the Ministry of Education and Science of Russian Federation.

\section{References}

Afanasiev, V. L., Amirkhanyan, V. R., Moiseev, A. V., Uklein, R. I., \& Perepelitsyn, A. E., SCORPIO-2 guiding and calibration system in the prime focus of the 6-m telescope. 2017, Astrophysical Bulletin, 72, 458, DOI: 10.1134/S1990341317040095

Amirkhanyan, V. R., BV RI observations of the radio source S5 0716+71. 2006, Astronomy Reports, 50, 273, DOI: 10.1134/S1063772906040020

Beers, T. C., Preston, G. W., Shectman, S. A., \& Kage, J. A., Estimation of Stellar Metal Abundance. I. Calibration of the CA II K Index. 1990, Astron. J., 100, 849, DOI: $10.1086 / 115568$

Burenin, R. A., Vikhlinin, A., Hornstrup, A., et al., The 400 Square Degree ROSAT PSPC Galaxy Cluster Survey: Catalog and Statistical Calibration. 2007, Astrophys. J., Suppl., 172, 561, DOI: 10.1086/519457

Covino, S., Baglio, M. C., Foschini, L., et al., Short timescale photometric and polarimetric behavior of two BL Lacertae type objects. 2015, Astron. Astrophys., 578, A68, DOI: 10.1051/0004-6361/201525674

Geyer, E. H., Jockers, K., Kiselev, N. N., \& Chernova, G. P., A novel quadruple beam imaging polarimeter and its application to Comet Tanaka-Machholz 1992 X. 1996, Astrophys. Space Sci., 239, 259, DOI: 10.1007/BF00645780

Gorshkov, A. G., Ipatov, A. V., Konnikova, V. K., et al., Rapid variability of the radio flux density of the blazar J0721+7120 (S5 0716+714) in 2010. 2011, Astronomy Reports, 55, 1096, DOI: 10.1134/S1063772911120043

Gorshkov, A. G., Ipatov, A. V., Konnikova, V. K., et al., Variability of the radio flux density of the Blazar S5 0716+714 on time scales less than a month. 2011, Astronomy Reports, 55, 97, DOI: 10.1134/S106377291102003X

Grossmann, A. \& Morlet, J., Decomposition of Hardy Functions into Square Integrable Wavelets of Constant Shape. 1984, SIAM Journal on Mathematical Analysis, 15, 723, DOI: $10.1137 / 0515056$

Impey, C. D., Bychkov, V., Tapia, S., Gnedin, Y., \& Pustilnik, S., Rapid Polarization Variability in the BL Lacertae Object S5 0716+714. 2000, Astron. J., 119, 1542, DOI: $10.1086 / 301286$

Larionov, V. M., Jorstad, S. G., Marscher, A. P., et al., The Outburst of the Blazar S5 0716+71 in 2011 October: Shock in a Helical Jet. 2013, Astrophys. J., 768, 40, DOI: $10.1088 / 0004-637 \mathrm{X} / 768 / 1 / 40$

Li, X., Mohan, P., An, T., et al., Imaging and Variability Studies of CTA 102 during the 2016 January $\gamma$-ray Flare. 2018, Astrophys. J., 854, 17, DOI: 10.3847/15384357/aaa5ac 
Marscher, A. P., Jorstad, S. G., D'Arcangelo, F. D., et al., The inner jet of an active galactic nucleus as revealed by a radio-to- $\gamma$-ray outburst. 2008 , Nature, 452, 966, DOI: $10.1038 /$ nature06895

Marscher, A. P., Jorstad, S. G., Larionov, V. M., et al., Probing the Inner Jet of the Quasar PKS 1510-089 with Multi-Waveband Monitoring During Strong Gamma-Ray Activity. 2010, Astrophys. J., Lett., 710, L126, DOI: 10.1088/2041$8205 / 710 / 2 /$ L 126

Moore, R. L., Angel, J. R. P., Duerr, R., et al., The noise of BL Lacertae. 1982, Astrophys. J., 260, 415, DOI: 10.1086/160266

Nilsson, K., Pursimo, T., Sillanpää, A., Takalo, L. O., \& Lindfors, E., Detection of the host galaxy of S5 0716+714. 2008, Astron. Astrophys., 487, L29, DOI: 10.1051/00046361:200810310

Oliva, E., Wedged double Wollaston, a device for single shot polarimetric measurements. 1997, Astron. Astrophys., Suppl., 123, 589, DOI: 10.1051/aas:1997175

Pryal, M., Falcone, A., \& Stroh, M., A Search for Fast X-Ray Variability from Active Galactic Nuclei Using Swift. 2015, Astrophys. J., 802, 33, DOI: 10.1088/0004$637 \mathrm{X} / 802 / 1 / 33$

Romer, A. K., Nichol, R. C., Holden, B. P., et al., The Bright SHARC Survey: The Cluster Catalog. 2000, Astrophys. J., Suppl., 126, 209, DOI: 10.1086/313302

Shablovinskaya, E. S. \& Afanasiev, V. L., The intraday variations of the polarization vector direction in radio source S5 0716+714. 2019, Mon. Not. R. Astron. Soc., 482, 4322, DOI: $10.1093 / \mathrm{mnras} / \mathrm{sty} 2943$

Stadnik, M. \& Romani, R. W., Imaging Redshift Estimates for Fermi BL Lac Objects. 2014, Astrophys. J., 784, 151, DOI: 10.1088/0004-637X/784/2/151

Stickel, M., Fried, J. W., \& Kuehr, H., The complete sample of 1 Jy BL Lac objects. II. Observational data. 1993, Astron. Astrophys., Suppl., 98, 393

Urry, C. M., Scarpa, R., O'Dowd, M., et al., The Hubble Space Telescope Survey of BL Lacertae Objects. II. Host Galaxies. 2000, Astrophys. J., 532, 816, DOI: $10.1086 / 308616$ 